\title{
Cooperativismo y Universidad
}

\author{
Siegbert Rippe
}

Sumario: I. Introducción. - II. Primera aproximación a la realidad cooperativo-educativa.- - III. La educación desde el punto de vista de las cooperativas. - IV. Desarrollo de los socios dentro de las cooperativas: la educación. - V. Educación Cooperativa.-VI. Universidad y Cooperativismo, los inicios. - VII. La realidad de las Cooperativas y la Universidad en la actualidad. —VIII. Conclusiones.

\author{
«Como afirmó Watkins, el movimiento \\ cooperativo es un movimiento económico \\ que hace educación» o «un movimiento \\ educativo que hace economía». \\ «La demanda del movimiento cooperativo \\ dirigido a la Universidad debiera ser \\ amplísima, cuantitativa y cualitativamente». \\ Francisco Vivent Chuliá
}

\section{Introducción}

La presente aportación a la temática en proceso de investigación se basa exclusivamente en la realidad que se conoce y en las perspectivas que se perciben en el relacionamiento entre Cooperativismo y Universidad, en Uruguay.

Es por ello también que se ha recurrido casi exclusivamente a bibliografía nacional, a fuentes de información igualmente uruguayas, entre las que se destacan entidades tales como la Confederación Uruguaya de Entidades Cooperativas (CUDECOOP) y la Unidad de Estudios Cooperativos (UEC) de la Universidad de la República, a la vez que se vuelcan algunas experiencias y vivencias del propio autor en la materia, en tanto que en ciertas épocas de su vida profesional fungió, en para- 
lelo con su actividad docente, como Asesor de la Dirección Nacional de Fomento Cooperativo del Ministerio de Trabajo y Seguridad Social y, por otro lado, como integrante de la Comisión Universitaria de Cooperativismo y como primer Coordinador de la citada Unidad universitaria (UEC).

Corresponde aclarar que la información universitario-cooperativa que se maneja en esta materia se relaciona exclusivamente con la vinculada con la estatal Universidad de la República, citada, que es la que, sin perjuicio de la segura, probable o eventual existencia y presencia de otros escenarios universitarios alternativos en la especie, exhibe de forma manifiesta y en los últimos 20 años una notoria e institucionalizada vocación de acercamiento y desarrollo plurisectorial en la temática planteada.

Corresponde agradecer asimismo la generosa colaboración recibida por parte de CUDECOOP y de la UEC, sin la cual no hubiera sido posible concretar razonablemente la presente investigación, a la vez que a la Lic. Karen Rippe por el desinteresado y eficiente apoyo brindado para la búsqueda, captación y organización iniciales de la información que nutre el presente reporte investigativo.

\section{Primera aproximación a la realidad cooperativo-educativa}

En Uruguay las cooperativas juegan un rol muy importante en la sociedad y su crecimiento ha sido permanente.

En efecto.

En el Relevamiento Nacional de Entidades Cooperativas de 1989 se constató la existencia de cerca de 900 cooperativas activas que agrupaban alrededor de 600.000 socios $^{1}$.

En el Relevamiento de Cooperativas de 1996 se determinó la existencia de 1264 estimadas con un total de 808.861 socios $^{2}$, y más próximo a la actualidad, y según estimaciones atribuidas al Gobierno nacional, existiría un total de 2.000 cooperativas, aproximadamente, que tendrían también un total estimado de 1 millón de socios, esto es, casi $1 / 3$ de la población uruguaya ${ }^{3}$. Lo cual supondría un crecimiento

\footnotetext{
1 Errandonea, Alfredo, Supervielle, Marcos: Las Cooperativas en el Uruguay, año 1992.

2 Bertullo, Jorge, Castro, Diego, Isola, Gabriel, Silveira, Milton: «El cooperativismo en Uruguay». Red Universitaria de las Américas en: Estudios Cooperativos y Asociativismo, año 2003.

3 Op. Cit. 2
} 
también aproximado del $60 \%$ de las unidades cooperativas y del $25 \%$ de los asociados, en el lapso de unos pocos, más recientes años, una información que no consta actualizada a la fecha.

Frente a esta realidad diferentes leyes y organismos estatales han creado instituciones y áreas especializadas y han desarrollado actividades de interrelacionamiento con las cooperativas, aunque las primeras formaciones cooperativas ya existen en Uruguay desde $1870^{4}$ y sin perjuicio de que desde entonces su vínculo con el Estado ha ido cambiando en su modalidad.

Desde avanzada la década del 80, siglo pasado, la promoción cooperativa en general estuvo inicial, formal y fundamentalmente centrada en la Dirección Nacional de Fomento Cooperativo del Ministerio de Trabajo y Seguridad Social, aun cuando en el Ministerio de Ganadería, Agricultura y Pesca (MGAP) operaba una unidad especializada en el relacionamiento con las cooperativas agrarias en particular, así como en el Ministerio de Vivienda (actualmente: de Vivienda, Ordenamiento Territorial y Medio Ambiente, MVOTMA), otra dedicada a la promoción de las cooperativas de vivienda en especial.

En la actualidad los vínculos entre las cooperativas y el Estado se establecen de manera parcialmente divididos entre diversas áreas, algunas que son de fomento, otras que son básicamente de contralor. Existe, sin embargo, desde marzo de 1991 la Comisión Honoraria del Cooperativismo que funciona en el ámbito formal de la Oficina de Planeamiento y Presupuesto (OPP), y está integrada por representantes estatales y de la Confederación Uruguaya de Entidades Cooperativas (CUDECOOP), la que tenía como fines o cometidos iniciales: velar por el cumplimiento de los principios cooperativos; promover y fomentar el desarrollo del movimiento cooperativo; asesorar y colaborar en la implementación de una política nacional; brindar asistencia técnica a las cooperativas; organizar un servicio estadístico y de difusión cooperativos; analizar y proponer reformas en materia del marco normativo.

Tales fines o cometidos se confirmaron, se ampliaron o se modificaron en el año 2005, año en el que se le establecieron los siguientes: "promover y fomentar el desarrollo de las cooperativas y del sector cooperativo en general, y brindar asistencia técnica a las cooperativas, coordinando su actividad con las cooperativas de grado superior; velar por el cumplimiento de los valores y principios cooperativos; asesorar en la fijación de la política nacional en materia de cooperativas y colaborar en su implementación coordinando con otros organismos oficia-

${ }^{4}$ Op. Cit. 2. 
les competentes, en su ejecución; informar a las autoridades competentes sobre los proyectos de ley y otras normas que incidan sobre las cooperativas; organizar un servicio estadístico y de información sobre cooperativas, pudiendo requerir información periódica y sistemática de las mismas, directamente o a través de sus organismos de integración. A estos efectos la referida Comisión puede coordinarse con los Registros Públicos correspondientes, la Auditoría Interna de la Nación y otros organismos públicos y analizar el marco normativo vigente en materia de cooperativas, y proponer la reforma que exige un concepto moderno de cooperativismo, así como la unificación de la regulación del tema en un solo cuerpo legal.

La misma disposición normativa habilitó adicionalmente a la mencionada Comisión, en el ejercicio de sus competencias, a coordinar su labor con otros organismos competentes por razón de las actividades de las cooperativas; proyectar el plan nacional de desarrollo cooperativo en coordinación con los representantes de los Ministerios competentes para cada rama, dando seguimiento a su ejecución; vigilar el correcto uso de la palabra «cooperativa», de acuerdo con la legislación vigente, así como de los símbolos del cooperativismo y denunciar ante las autoridades competentes los casos de apartamiento de dichas normas; requerir información periódica y sistemática a las cooperativas, directamente o a través de sus organismos de integración, al efecto de la prestación de su servicio estadístico y de información general; en general, velar por el estricto cumplimiento de las disposiciones legales vigentes, cuidando de no entorpecer la regular administración de las cooperativas; ejercer la representación estatal nacional en la Reunión Especializada de Cooperativas del MERCOSUR (RECM); y en general, adoptar todas las medidas necesarias, dentro de su competencia, para el cumplimiento de sus cometidos.

La solución promocional-institucional arriba referenciada, que muestra la voluntad estatal de instrumentar una mayor y mejor satisfacción a las diversas y crecientes necesidades del sector cooperativo, no sustituyó en su contexto específico las prestadas por el MGAP y el MVOTMA, citados, a la vez que en 2006 se reguló la relativa a las llamadas «Cooperativas Sociales», en la órbita gubernamental del Ministerio de Desarrollo Social, de nueva y más reciente creación institucional.

Corresponde señalar que se encuentra en estado parlamentario un proyecto de ley general de cooperativas que prevé una suerte de Consejo Superior de Cooperativismo, con integración de representantes del Estado y del movimiento cooperativo organizado en la CUDECOOP, que asumiría las tareas promocionales otorgadas actualmente a la citada Comisión Honoraria de Cooperativismo. 
En términos operativos cada modalidad cooperativa está también y particularmente sometida a mecanismos de contralor por parte del Estado, los que están mayormente centralizados en una llamada "Auditoría Interna de la Nación», organismo desconcentrando dependiente del Ministerio de Economía y Finanzas, aunque en algunos casos operan dividida o compartidamente en la materia con otras unidades público-administrativas de control.

No obstante, no se advierte, salvo tal vez en tiempos más recientes, un conocimiento más orgánico y una respuesta más consistente del Estado en la materia cooperativa. Así, por ejemplo. En el '97 se realizó un trabajo donde la Universidad del la República fue partícipe y en el que se constató que en los catorce (14) organismos públicos relevados en la oportunidad, la variable cooperativa no había sido ciertamente considerada o establecida, lo cual significó una faltante de datos estatales confiables, necesarios o relevantes vinculados al sector cooperativista.

Sin embargo, y puntualmente, a fines de 1999 se creó una comisión especial que trataría el tema de las relaciones laborales en las cooperativas. Esta Comisión funcionaría en el ámbito del Ministerio de Trabajo y Seguridad Social, invitándose a participar también a representantes de la Oficina de Planeamiento y Presupuesto, del Ministerio de Economía y Finanzas y del Banco de Previsión Social.

Mientras tanto la citada CUDECOOP, órgano superior del movimiento cooperativo organizado, integra varios ámbitos de relacionamiento con organismos públicos, como por ejemplo: Comisión Social Consultiva de la Universidad de la República y el Grupo promotor por el Desarrollo de Montevideo (que está formado por las Cámaras de Industrias y de Comercio y Servicios, Intendencia Municipal de Montevideo, la Universidad de la República y la Universidad ORT, entre otros).

En tal contexto administrativo-funcional, la educación formal en el sector cooperativo, a nivel estatal y en su externalidad, sin perjuicio de algunas excepciones en la enseñanza primaria y media y de los destacables y puntuales esfuerzos intra e intercooperativos, luciría en primera instancia relativamente relegada por los objetivos primarios del cooperativismo, que están relacionados prevalentemente a lo económico-social y a lo laboral.

Sin embargo, estos cometidos sí son parte de la relación entre la Universidad y el cooperativismo, sobre todo aquéllos relativos a la promoción y fomento del desarrollo cooperativo, al asesoramiento y a la colaboración con las cooperativas y la difusión de las experiencias cooperativistas en general. 


\section{La educación desde el punto de vista de las cooperativas}

Las cooperativas fueron creadas con un fin socio-económico, sin embargo, dentro de sus principios fundamentales existe el «Principio de la Educación». Esto significa o más bien se refleja en la constitución de un fondo para la educación, sea a nivel de una cooperativa de primer grado, sea a nivel de una cooperativa de segundo o ulterior grado.

Esto es. Si bien la cooperativa tiene una finalidad económica y social lo hace mediante una organización que tiene dentro de sí misma, dentro de su propia finalidad, la de educar y formar al socio.

La idea es resolver la necesidad socio-económica del socio a través de una organización económico-social que además de solucionar el problema económico brinde al socio la educación y la formación. Es aquí donde la educación y su vínculo con las universidades juega un rol fundamental dentro de los objetivos cooperativos.

Esto es. La educación y su fomento van más allá de la pura actividad económica o social y se centra en el desarrollo de los socios y de sus posibilidades.

\section{Desarrollo de los socios dentro de las cooperativas: la educación}

Como se vio anteriormente las cooperativas tienen primordialmente un fin económico no exento de un fin social, pero también promueven, fomentan y buscan mejorar y brindar oportunidades educativas a sus recursos, componentes o integrantes humanos. El aprendizaje, por ende, va más allá de lo sólo laboral.

Las cooperativas quieren y necesitan crecer y desarrollarse, para ello es también fundamental contar con socios cada vez más especializados en sus áreas de desempeño. Aunque muchas veces la solución inmediata para seguir creciendo significa para las cooperativas postergar el desarrollo del aprendizaje, ya sea por problemas económicos, por tiempo o por falta de recursos.

Las cooperativas, muchas veces de forma teórica pero no tan práctica saben que la optimización de recursos, el crecimiento de la propia cooperativa y la búsqueda de nuevas oportunidades, son el resultado de un personal cada vez más profesionalizado y educado acorde a sus objetivos.

\section{Educación Cooperativa}

La Educación Cooperativa o la Formación cooperativa significa: 
1. Todo lo que esté orientado a las actividades que tengan como fin el acercamiento de nuevas personas a la organización cooperativa, con el fin de aumentar la escala de la organización.

2. Las actividades que se realizan dentro de la cooperativa, que signifiquen que las personas que ya son socias se mantengan integradas e interesadas por la actividad cooperativa.

3. La designación de actividades dirigidas a los dirigentes, colaboradores y demás con el fin de aumentar las capacidades de los mismos ${ }^{5}$.

El vínculo con la Universidad, sus cursos, talleres, conferencias, seminarios, especializaciones y demás eventos pueden ayudar en, por un lado, incentivar la integración de los ya socios en las cooperativas y por otro, la capacitación dirigida a elevar el nivel de conocimientos de dirigentes y colaboradores.

Según la Asistente Social Zaffaroni de Aguiar, ${ }^{6}$ citada, la capacitación en las organizaciones cooperativas debe contar con:

1. Creación de un plan general a partir de necesidades específicas. La capacitación debe fomentarse de manera integrada y con un plan de acción de la misma. Esto significa capacitar en los cursos adecuados, de la forma adecuada al personal adecuado. Los cursos deben ser con el fin de llevar adelante los objetivos de la cooperativa, para su desarrollo y optimización. Además se debe tener en cuenta la posibilidad de retransmisión de estos cursos al resto de los integrantes de las cooperativas.

2. Los contenidos de la capacitación. Es importante tratar de capacitar en todas las áreas de las cooperativas, esto incluye los sectores de: gerenciamiento y administración, del área específica a la que se dedica la cooperativa y los cursos vinculados a la dinámica e interrelacionamiento de los grupos.

3. Los instrumentos. Esto se refiere a la metodología y a las herramientas para desarrollar de forma óptima los programas de capacitación. Esto significa el material, los días de la capacitación, los docentes, etc.

4. Las características de un programa. El programa debe ser sistemático y planificado, con objetivos concretos y que pueda implementarse. Además los programas deben ser adecuadamente dirigido a quienes participaran en él. Todos los programas deben poder evaluarse.

5 ZafFARONI DE AgUIAR, Cristina; «Integración y Educación Cooperativa», del libro Cursillo sobre cooperativismo de la Universidad de la República, ed. FCU, mayo 1988.

6 Op. cit. 5 


\section{Universidad y Cooperativismo, los inicios}

El acercamiento de las cooperativas y la Universidad en la década del '80

A partir de 1985, con la recuperación de la democracia en el país y en particular con la recuperación de la Universidad de la Republica, el Estado redefinió acciones institucionales con el fin de acoplar la Universidad a la realidad socioeconómica y política uruguaya. La Universidad debía tener como uno de sus fines ser un actor principal que vinculara la realidad con los planes de estudios y acciones universitarias.

La observación y el ser parte de la realidad le permitió a dicha Universidad estudiar el fenómeno cooperativista que se venía gestando y las posibilidades de crecimiento y perspectivas del mismo dentro de la realidad nacional. Las cooperativas eran advertidas como un mecanismo idóneo de solución de las necesidades de la población.

Este hecho hizo que la Universidad de la República se posicionara como un actor social e institucional que favoreciera el desarrollo y desempeño de este movimiento cooperativo.

Con este fin se siguieron tres primarios caminos simultáneos e interrelacionados:

1. Se sistematizó en el ámbito interno de la Universidad las actividades que se realizaban vinculadas al sector cooperativo, en el espacio de sus tres funciones legales básicas, Docencia, Investigación y Extensión; y se estableció un vínculo institucional con el sector, a través de CUDECOOP, así como una relación también institucional con las dependencias del sector público responsables del contralor y la promoción de las entidades cooperativas.

2. Con el objetivo de llevar adelante una gestión exitosa se creó a través del Consejo Directivo Central de la Universidad de la República la Comisión Universitaria de Cooperativismo, integrada por representantes de todas las dependencias que se vinculaban al sector o podían desarrollar actividades con el sector cooperativo de manera interinstitucional y multidisciplinaria.

Durante esta época se firmaron Convenios de cooperación técnica y científica con el Ministerio de Trabajo y Seguridad Social (MTSS) y con la CUDECOOP. Además, la mencionada Universidad desarrolló un curso de actualización docente en cooperativismo.

3. El tercer camino es el vinculado a las relaciones internacionales entre las universidades y el tema del cooperativismo. En función de esto se establecieron vínculos y se desarrollaron actividades con la Asociación Latinoamericana de Centros de Educación Cooperativa (ALCECOOP), con el Instituto de Cooperación Internacional (ICI) y con el IL- 
PES-CEPAL, entre otros. Por su parte, la misma Universidad buscó la institucionalización de acciones vinculadas a las cooperativas.

Bajo esta consigna la Universidad participó de tres formas principales:

a. El Primer Relevamiento Nacional de Entidades Cooperativas. El mismo fue el resultado de la intercooperación institucional entre la Universidad, el MTSS y el sector cooperativo ya entonces nucleado en la CUDECOOP. A su vez, el apoyo financiero para la realización del mismo provino de la Organización de Estados Americanos (OEA). Aquel permitió en el año 1989 obtener información relevante y actualizada del sector cooperativo a nivel nacional, tanto en sus aspectos cuantitativos básicos así como en su dimensión propiamente social.

b. Una investigación sobre la incidencia del Cooperativismo en la economía nacional y cómo se insertan las cooperativas en el mismo, ello, en el marco de un Convenio celebrado por la Universidad y la CUDECOOP, su unidad ejecutora es el Instituto de Economía de la Facultad de Ciencias Económicas y Administración. Además del comportamiento económico de las entidades cooperativas y del análisis de las principales variables, este trabajo posibilitó un análisis global del impacto socioeconómico del sector en el largo plazo y la evaluación sobre sus potenciales líneas de desarrollo.

c. La creación de la Unidad de Estudios Cooperativos (UEC). Esta Unidad tiene por objetivos programar, coordinar, evaluar y realizar actividades de docencia, investigación y extensión universitarias en el área del cooperativismo, tanto a la interna de la Universidad como a la externa de la misma. A su vez, se relaciona de manera funcional como Secretariado.

La UEC buscó diferentes instancias de participación y colaboración. Una de las más importantes por sus resultados fue el «Cursillo de Cooperativismo» realizado en 1987 y que fuera luego publicado por la Fundación de Cultura Universitaria, editorial uruguaya de primera línea en lo jurídico, contable, económico y social, en dos librillos (Tomos I y II).

Durante el cursillo los expositores (docentes, profesionales y cooperativistas) expusieron las necesidades y los vínculos posibles entre las cooperativas y la Universidad.

Más allá de las áreas específicas de cada cooperativa hubieron, puntos de sinergia que pueden resumirse en los siguientes: 
1. Promover y difundir trabajos, monografías e investigaciones que se realizaron de las cooperativas a las cooperativas en sí mismas y a la sociedad en general con el fin de aprender de otras experiencias, conocer puntos de vista que fomenten el trabajo de las cooperativas e integrar el cooperativismo a la sociedad en general.

2. Promover la investigación de las cooperativas en varias materias, no sólo en aquellas vinculadas al objetivo mismo de la cooperativa sino que también de su estructura, administración, psicología, recursos humanos, comercialización, etc.

3. Interrelacionar las investigaciones a nivel universitario. Como por ejemplo: si hay una cooperativa de vivienda, la Facultad de Arquitectura puede estudiar su diseño, sus características habitacionales y estructura, mientras que de esa misma cooperativa la Facultad de Ciencias Económicas y de Administración estudia su gestión, administración y contabilidad y la Facultad de Medicina, por su parte establece la posibilidad de integrar centros de salud, control sanitario y servicios médicos.

4. Investigaciones para fomentar a las cooperativas. Esto se refiere tanto a una perspectiva de generar más integración, nuevos socios y un mayor desarrollo de las cooperativas, así como también para fomentar a otras instituciones a colaborar con las cooperativas (por ejemplo, Bancos, asociaciones. Industrias, etc.).

5. Cursos, seminarios, conferencias, etc. para los nuevos cooperativistas de cómo desarrollar una cooperativa de cómo integrarse para salir adelante de situaciones complejas y/o para fomentar la creación de nuevas cooperativas.

6. Las Universidades deben además fomentar en sus cursos regulares el desarrollo cooperativo y las posibilidades de las cooperativas de ser parte de la estructura de la sociedad. Los cursos más que ser dispersos y separados deberían ser concentrados dentro de las posibilidades económicas de la Universidad. Por ello la importancia de los equipos interdisciplinarios y multidisciplinarios.

7. Las Universidades pueden apoyar el funcionamiento de la gestión cooperativa a través de las consultorías en todas sus áreas que permitan abordar, esclarecer, prevenir y mejorar el desempeño de las cooperativas y su capacidad de crecimiento. Esto también implica el enseñar alternativas tanto del mercado interno como externo para que cada área pueda tener una planificación acorde a su capacidad e inter relacionada con las otras áreas y el mercado. 
8. Creación de un Instituto Universitario que se dedique a abordar los temas cooperativos en su conjunto a través de cursos de posgrado con el fin de conocer y manejar las peculiaridades del funcionamiento de una cooperativa.

9. Las cooperativas deben y pueden actuar en conjunto a través del intercambio y la cooperación, para ello el rol de la Universidad puede ser el de fomentar dicha integración desde un punto educativo pero también empresarial y directivo.

10. La integración de docentes, universitarios y docentes a los sistemas cooperativos como práctica y como teoría.

11. En el campo de la extensión se puede lograr que entre el intercooperativismo y las relaciones interregionales de la Universidad, se fomente y difunda el posicionamiento de las cooperativas en sus respectivos sectores. Esto significaría un posicionamiento de las cooperativas en el mercado. A través de la extensión se pueden también hacer esfuerzos de cooperación en lo que se refiere a la tecnología, su generación y transferencia.

12. La Universidad y sus diferentes facultades pueden también evaluar el sistema cooperativo y cada cooperativa, como auditores externos.

\section{La realidad de las Cooperativas y la Universidad en la actualidad}

Durante éstas últimas dos décadas la Universidad y las Cooperativas han llevado adelante caminos en conjunto con el fin de llegar a los objetivos establecidos 20 años atrás. Dentro de las acciones que se llevaron a cabo, en función del cursillo y de los objetivos de las cooperativas y las universidades, se logró avanzar en los siguientes ámbitos:

\section{La UEC y su rol}

a. La Unidad de Estudios Cooperativos en el año 2002 redefinió sus objetivos, ya que se observaba que hasta entonces había una falta de dinámica entre los objetivos y la realidad. Esto significó la consolidación de un grupo multidisciplinario de trabajo que incluye docentes, estudiantes y universitarios que realiza consultorías para las cooperativas. Este grupo ha venido en crecimiento desde entonces. 
b. La Unidad de Estudios Cooperativos del Servicio Central de Extensión y Actividades en el Medio, desde el 2003, realiza un curso de grado de "Cooperativismo y Procesos Asociativos» que está dirigida a todos los estudiantes universitarios.

c. La UEC decide crear un tipo de organización estructural similar a las de una Cooperativa, donde existen 2 ámbitos de decisión: un plenario y un equipo coordinador.

d. La UEC así se desarrolla en varias ramas:

i. Formación: Realización de cursos sobre Cooperativismo y asociativismo, por un lado, y por otro, instancias de formación de grupos cooperativos y pre-cooperativos.

ii. Incubadora: Denominan de esta forma a los estudios que realizan buscando con las cooperativas las posibilidades de viabilidad social y económica de su sector. Esta rama colabora tanto con cooperativas ya formadas como con cooperativas en formación.

iii. Investigación: Se centran en diversos temas relativos al desarrollo de las cooperativas en el Uruguay y exponen tanto a nivel nacional como internacional, intercambiando información con universidades privadas. Un claro ejemplo fue la investigación acerca de: «Impacto de la integración regional del MERCOSUR sobre el sector cooperativo», en la que participaron Universidades de Chile, Paraguay, Argentina, Colombia y Brasil, además de la propia Universidad de la República, de Uruguay. Este seminario que se realizó en junio de 2007, tuvo un fuerte impacto en estudiantes y cooperativas.

iv. Grupo de estudio: Este grupo se subdivide en varias áreas, ellas son: Cooperativas de Trabajo y empresas recuperadas, Cooperativas Agrarias y Desarrollo Rural, Cooperativas de Vivienda, Pedagogía y Formación Cooperativa, Modelos de gestión y Organización del trabajo y Políticas Públicas y Cooperativas.

e. La revista de estudios cooperativos. Esta revista se divide en secciones donde se tratan temas tales como:

i. Las investigaciones y su divulgación (las mismas son seleccionadas por el tipo de estudio, por los aportes e innovaciones que realiza a nivel cooperativo).

ii. Otra sección es la relativa a publicar los trabajos y las investigaciones de los estudiantes universitarios.

iii. Una tercera sección que tiene como fin promover a través de artículos a los actores cooperativos. 
iv. Una cuarta y última sección que tiene artículos referentes a las notas de divulgación académica.

Esta revista busca fomentar el intercambio de información, y con este fin hace llamados abiertos a docentes, estudiantes, egresados y cooperativistas, a participar y publicar sus artículos y/o estudios.

f. La UEC también lleva adelante desde el 2003 un curso para estudiantes de grado relativo al Cooperativismo y asociativismo, con el fin de los mismos se adentren más en el mundo del cooperativismo. Este curso es accesible para todas las carreras de grado de la Universidad de la República.

g. La investigación científica y el cooperativismo. Esto es directamente relativo al vínculo entre la Universidad y las Cooperativas ya que la mayoría de las investigaciones son realizadas en las facultades. Esto es porque la Universidad cuenta con tres mecanismos de financiación para esta área:

i. La financiación por fondos concursables centrales de la Universidad,

ii. La financiación de horas de investigación por la vía del Servicio (dependencia universitaria) a la cual pertenece el investigador, y;

iii. Los Convenios entre el investigador y los sectores público y privado.

Respecto de la investigación generada a partir de fondos centrales de la Universidad, existe la Comisión Sectorial de Investigación Científica (CSIC) cuya finalidad es justamente el fomento integral de la investigación en la Universidad de la Republica.

Para llevar adelante su fin la CSIC tiene programas vinculados con:

- Dedicación Total,

- Proyectos de Investigación y Desarrollo,

- Fortalecimiento Institucional,

- Proyectos de Vinculación con el sector productivo,

- Recursos Humanos,

- Iniciación a la Investigación, y

— Formación de Postgrado.

2. Los convenios entre las Cooperativas y la Universidad y su aplicación en las diferentes facultades

a. Departamento de Ciencias Sociales de la Facultad de Agronomía. Desde fines de la década del 80 realiza un curso de Coope- 
rativismo Agrario. Por el momento es el único curso específico de cooperativismo de toda la Universidad.

b. Las Facultades de Psicología y de Ciencias Sociales tienen varios convenios con cooperativas para pasantías de estudiantes. Además, la Facultad de Ciencias Sociales colaboró en el Censo de Cooperativas que se realizó en 2000.

c. En lo que se refiere a la Facultad de Derecho, existen dentro de su plan general varios materias con contenidos programáticos vinculados al Derecho Cooperativo, y una reciente Cátedra de Derecho Cooperativo para el desarrollo de una materia optativa de grado, inserta administrativa y funcionalmente en el ámbito formal del Instituto de Derecho Comercial de esa Facultad, sin perjuicio de prever una participación docente multidisciplinaria. Existe, asimismo, intención de proyectar un posgrado en tal rama del Derecho, sola o inserta en un esquema de posgraduación de mayor amplitud y alcance, vinculado en este caso con las formas y organizaciones jurídico-sociales asociativas no lucrativas.

d. Por su parte en la Facultad de Ciencias Económicas y de Administración existen varias consultorías y pasantías en cooperativas para estudiantes y egresados universitarios.

e. A finales del año 2004 la Universidad de la República creó una Red Temática sobre Proceso Asociativos donde se inter relacionan grupos académicos de varias facultades que están interesados en el cooperativismo.

f. En el año 2001 las Jornadas Universitarias sobre Cooperativismo tuvieron una amplia convocatoria. Estas Jornadas tuvieron como propósito difundir las actividades y los objetivos por los cuales la UEC fue creada.

g. En la Licenciatura en Trabajo Social de la Facultad de Psicología también se realizan prácticas pre-profesionales con el fin de que los estudiantes aprendan en una cooperativa cómo se realiza dicho trabajo y la realidad de su futura profesión.

3. El punto de vista de las cooperativas en su relación con la Universidad

Desde el punto de vista del movimiento cooperativo es notorio el interés del mismo por los avances en materia de investigaciones y cursos de y para el Cooperativismo, así como la disposición de diferentes agentes de las cooperativas a colaborar y participar en cursos, seminarios, investigaciones y formación de los mismos universitarios. 
Sin embargo, 20 años después del Cursillo realizado en 1987, oportunamente citado, las cooperativas siguen advirtiendo algunos problemas y falencias con respecto a la Universidad7:

1. La escasa formación que aún existe hoy en casi todas las carreras vinculadas a las posibilidades del desarrollo de las cooperativas. Por ello consideran pertinente llevar adelante más acciones de capacitación del área y especialmente del funcionamiento, el rol y las posibilidades que representa en la sociedad una cooperativa.

2. Además, solicitan investigaciones, consultorías y análisis concretos de la realidad actual en lo que se refiere a las cooperativas y su rol en la sociedad y en las políticas estatales a nivel nacional e internacional.

3. Otro tema que aún tiene falencias es el propio fortalecimiento del vínculo entre la Universidad y las cooperativas.

\section{Las Universidades y el Cooperativismo a nivel internacional.}

a. La Red Universitaria de las Américas (de la cual Uruguay es uno de sus miembros) busca precisamente estrechar, afianzar y promover el vínculo entre los sujetos de la universidad y el movimiento cooperativista a nivel internacional. A través de la Red Universitaria de la Américas se ha logrado facilitar el conocimiento y la formación desde una perspectiva regional e internacional, impulsando a las cooperativas a mejorar e intercambiar capacidades que le ayuden a asegurar el éxito en su trabajo social y económico en el medio en el que se desenvuelven.

b. Además se coordina el Comité Académico sobre Procesos Cooperativos y Entidades Económicas Asociativas (PROCOAS) de la Asociación de Universidades del Grupo Montevideo, siendo referencia académica en el plano regional a través de la Reunión Especializada en Cooperativas de MERCOSUR (RECM).

c. Además, desde 1976, Uruguay pertenece a la ALCECOOP Asociación Latinoamericana de Centros de Educación Cooperativa, que surge con el compromiso de centros especializados de Argentina, Brasil, Chile, España y Uruguay con el fin de intercambiar experiencias.

7 Op. Cit 2. 
d. También existe la AGITCOOP, Comité Ejecutivo del Grupo Asesor para la Capacitación Internacional de Cooperadores de la Alianza Cooperativa Internacional.

e. Otra fundación para educar e intercambiar experiencias y conocimientos es la FEOLAC, Fundación creada por la Confederación Latinoamericana de Cooperativas de Crédito.

\section{Conclusiones}

Las cooperativas pueden y deben fomentar la educación y para ello tienen una fuente tan importante y especializada como es la Universidad, sus cometidos y sus diferentes, específicas áreas de estudio.

La cantidad de cooperativas ya existentes y en constante aumento en Uruguay hace que las mismas sean un importante objeto de atención y estudio para las universidades, faltando aún procesos integratorios más fluidos.

En este país se observa, por lo pronto, una real y significativa interacción real entre la Universidad de la República, las cooperativas y los actores sociales conexos o afines que componen el escenario de colaboración y coordinación internacional en la materia; intercambios que fortalecen los vínculos entre universidades, sus sujetos y organizaciones sociales y proactivas al cooperativismo, y habilitan la reciprocidad en el conocimiento y la comunicación de las experiencias cooperativas.

Sin embargo, según CUDECOOP8, tanto la Comisión Honoraria de Cooperativismo como la Universidad tienen vínculos que lucen aún escasos con las cooperativas, e incluso señalaron que: "La función de promoción (referida a la Comisión mencionada) nunca se ha cumplido sistemáticamente, no existiendo ningún otro órgano específico para ejecutarla (...) se requiere (...) de información básica para decidir áreas a profundizar (datos estadísticos) y la vinculación con el sistema educativo (...) supone una coordinación entre los distintos Ministerios y organismos públicos».

Con referencia a la Universidad, en el año 2005 CUDECOOP señaló en «Las Segundas Jornadas Universitarias sobre cooperativismo, economía solidaria y procesos asociativos» que aquella debe cumplir «un rol más activo en la formación y promoción de investigación». ${ }^{9}$

${ }^{8}$ Bertullo, Jorge, CAstro, Diego, Isola, Gabriel, Silveira, Milton: «El cooperativismo en Uruguay». Red Universitaria de las Américas en, Estudios Cooperativos y Asociativismo, año 2003.

9 http://www.cudecoop.coop/novedades_archivo.htm 
En Uruguay se advierte que las cooperativas son progresivamente cada vez más numerosas, a la vez que su objeto y actividades comprenden la más heterogénea variedad de sectores y rubros económicos y sociales: Cooperativas de Consumo, Cooperativas de Ahorro y Crédito, Cooperativas de Producción y Trabajo Asociado, Cooperativas de Vivienda, Cooperativas Agrarias, Cooperativas Agroindustriales y Cooperativas Sociales. Esto significa, por su parte, que todas las unidades de docencia, investigación, extensión y asistencia que integran el universo de competencia funcional universitaria tienen la aptitud institucional necesaria para participar de una forma u otra, singularmente y particularmente en conjunto y multi e interdisciplinariamente, en temas y asuntos vinculados con sus respectivas especialidades, para fomentar las cooperativas, su desarrollo y sus intercambios.

El cooperativismo tiene un destacable carácter social y económico y las universidades pueden claramente ayudar en ambas. Desde el punto de vista social ofrece una alternativa doctrinario-organizacional que entusiasma y moviliza a sectores varios, tanto del gobierno como de la sociedad civil. Aquí nacen, se forman y desarrollan redes y relaciones solidarias que crean un entramado de mayor potencial económico y mejores perspectivas sociales.

Desde el punto de vista económico, por su parte, propone una alternativa teórico-práctica empresarial que basada en valores y principios de autogestión y ayuda mutua reinvindica y jerarquiza el factor humano en la realización de actividades organizadas de producción, prestación e intercambio de bienes y servicios y su incidencia personal en la satisfacción de sus necesidades de empleo o de consumo. Aquí, por su lado, se generan y desenvuelven emprendimientos idóneos a esos propósitos, de base sustantiva e instrumental igualmente solidaria, aptos para insertarse y contribuir en procesos de integración económico-cooperativa horizontal y vertical, en el espacio nacional, regional e internacional.

¿Cómo pueden contribuir en ello las universidades? Pues mucho y de varios modos, y la Universidad de la República es esencial, sin que ello implique negar o discriminar el aporte, importantísimo, que puedan efectuar las universidades privadas.

A título de ejemplos:

1. En un área de tanta importancia como es la formación cooperativa, las universidades pueden jugar un papel fundamental en el proceso de enseñanza-aprendizaje ya que cuentan con los recursos humanos, además de los materiales y tecnológicos necesarios para ello, además de que los mismos se pueden interrelacionar entre sí de forma orgánica, coordinada y permanente, de manera institucional o con 
base en proyectos educativos multi e interdisciplinarios, que permitan, por un lado, introducir el perfil cooperativo, integral o parcial, según las circunstancias del caso, en el grado y en el posgrado universitarios y, por otro lado, el desarrollo de servicios de capacitación para las cooperativas, sus asociados y dirigentes, a brindar de forma integrada, específica, metódica, medible y evaluable.

Incluso, la participación de cooperativistas en la propia elaboración de programas de cursos a realizar por las universidades con ingredientes cooperativos, puede fomentar procesos de socialización idóneos para crear e instrumentar nuevas estrategias de trabajo educativo en común, acceder al conocimiento de experiencias concretas, detectar necesidades reales a responder, y mejorar la calidad del vínculo entre cooperativistas y docentes.

Ello de alguna manera ocurrió - y ocurre - tanto a nivel universitario como cooperativo en Uruguay, en el que se busca desde hace más de dos décadas el fomentar aquel interrelacionamiento educativo y optimizar el respectivo funcionamiento institucional en esa área del conocimiento y de la realidad.

Una aclaración. El cooperativismo forma parte del programa de sexto año de la educación primaria, además, la educación cooperativa es parte de la Unidad de Educación Moral y Cívica (liceal) y se imparte en los Centros de Educación Técnico-Profesional. La Universidad de la República en especial, lo que hizo y hace $-\mathrm{y}$ lo hacen o pueden hacerlo otras universidades - es acompañar y complementar dicha educación a nivel de su competencia terciaria y cuaternaria.

En el país la UEC ha intentado desarrollar varias actividades de ese tenor, pese a que muchas son o lucen esporádicas. Sin embargo, consta - y así debe reconocerse y difundirse - que se ha focalizado en buscar una mayor sinergia entre la Universidad y las cooperativas a través de su participación directa en cursos específicos sobre temas relativos y/o de interés para las cooperativas, estudiantes, egresados y docentes, así como también en la recepción y posterior derivación de las diferentes demandas cooperativas, a las facultades y a la Universidad en su conjunto.

2. En un área metodológica y creativa de conocimientos como es la investigación corresponde reconocer que en el país el número de investigaciones universitarias relacionadas con el universo cooperativo es sin duda bajo, hecho que sorprende si se recuerda que cerca de un tercio de la población integra o participa de alguna cooperativa. Sin embargo, se destaca la participación del área agraria de la Universidad de la República (Agronomía y Veterinaria) en el desarrollo de investigaciones vinculadas al sector cooperativo. Esto puede deberse, por un lado, 
a la trascendente importancia económica que en términos generales posee el sector agropecuario y en éste, la modalidad cooperativa (cooperativas agrarias), y por otro, al desarrollo específico que el cooperativismo como tal posee en el ámbito académico de esa área, lo anterior, sin perjuicio de que pueden existir trabajos de tesis o simplemente monografías hechas en particular por estudiantes del grado.

En términos globales, pese a lo escaso de la información disponible y captada, puede afirmarse que la investigación en el mundo de lo cooperativo es o aparenta ser deficitaria en relación al potencial que posee la Universidad como un actor pertinente y con capacidad de aporte a la resolución de diversas problemáticas que poseen las cooperativas uruguayas en la actualidad. De todas formas se evidencian avances relativos en el plano de la investigación, con incidencia en la formación cooperativa y en el fenómeno de las empresas que habiendo estado en severas crisis fueron luego recuperadas.

Lo que no puede discutirse es que la investigación es un cometido esencial de toda universidad, que el análisis crítico de la realidad y la realización de propuestas posibles de cambio y transformación es una obligación universitaria de primer orden, que las cooperativas son o pueden ser agentes demandantes de tales investigaciones y que la Universidad tiene los instrumentos y mecanismos necesarios para dar respuesta a tales demandas.

3. En el campo de la extensión, eventualmente de la asistencia universitaria, la presencia y participación de las universidades son decisiones y acciones institucionales convenientes, si no necesarias, para apoyar la resolución de los problemas de la gente mediante el uso y aplicación de mecanismos e instrumentos calificados a disposición de aquellas, y no escapan a tal consideración las cooperativas como destinatarias finales de las mismas, por el contrario, son -y deben serusuarias y hasta preferenciales de esos mecanismos e instrumentos, en la medida que la ideología teórico-práctica que sustenta a esas organizaciones sociales y económicas, hecha de valores y principios, es compatible y afín con la propia de una comunidad humana que procura mejorar su calidad de vida y contribuir a la construcción de un país, sobre la base de una actitud más activa, democráticamente participativa, social y económicamente responsable y comprometida y éticamente solidaria de su gente.

El relacionamiento Universidad-Cooperativismo se inscribe naturalmente en el marco de la extensión universitaria, se ha manifestado, por otra parte, en la Universidad de la República, en múltiples y concertadas acciones que muestran el recíproco interés y voluntad en ampliar, profundizar y fortalecer tales relaciones, así como en la convicción de su nece- 
sidad, y permite concluir que el desarrollo de ese campo de la actividad universitaria, con acento, en la oportunidad, en la colaboración con el cooperativismo y las cooperativas amerita, justamente, su acentuación.

En el ámbito de esa misma Universidad es advertible la importancia que se atribuye a ese cometido universitario, cuando se recuerda que su Unidad de Estudio Cooperativos (UEC) se ubica en un Servicio administrativo-funcional dedicado, efectivamente, a la extensión y a las actividades en el medio, un mero ejemplo de lo que es posible - y seguramente deseable - en la materia.

En definitiva. Los hasta expuestos resultados de la investigación realizada sobre el tema en cuestión permiten concluir: en la importancia del relacionamiento institucional entre Universidad-Cooperativismo, y tanto en materia de docencia como de investigación y extensión; en la validez de las experiencias realizadas a nivel, por lo menos, de la Universidad de la República y del movimiento cooperativo del Uruguay; en la necesidad de incrementar y mejorar esas relaciones; en la conveniencia de intercambiar experiencias vividas, tanto en el país como en el exterior; en la también conveniencia de acceder y evaluar éxitos y fracasos, logros y frustraciones, de aprender de unos y de otros, de trabajar de manera coordinada, conectada y comprometida entre actores sociales tan diversos; de contribuir al mejor cumplimiento de los objetivos propios pero confluyentes de cada uno de ellos, de aportar, en fin, al esfuerzo común por un mundo más educado y más cooperativo.

\section{Bibliografía}

AANV, Cursillo sobre Cooperativismo de la Universidad de la República, Vol. 1 y 2 ed. 1988, Fundación de Cultura Universitaria (FCU)

-, Revista Boletín de Estudios Cooperativos UEC, Universidad de la República, año I, N. ${ }^{\circ}$ 1, junio 1990.

- Revista Estudios Cooperativos, UEC, Universidad de la República, Año 10, Num.1, febrero 2005.

- Revista Estudios Cooperativos, UEC, Universidad de la República, Año 12, Num. 1, abril 2007.

Bertullo, Jorge, Isola, Gabriela, CAstro, Diego, Silveira, Milton, «El Cooperativismo en Uruguay», Red Universitaria de las Américas, en Estudios Cooperativos y Asociativismo, abril 2003.

Mederos, Jorge, Los Nuevos Desafíos. El cooperativismo y la globalización, ed. CEDECOOP / Centro Cooperativo Sueco, 1998.

ERrandonea, Alfredo, Supervielle, Marcos, Las Cooperativas en el Uruguay. Análisis Sociológico del Primer Relevamiento Nacional de Entidades Cooperativas, ed. FCU, 1992. 
AANV, Jornadas de Estudio sobre Universidad, Cooperativismo y Economía Social (Segovia 1984), MTSS, Madrid 1986.

Sitios web

www.mercosur.coop.org

www.cudecoop.coop

www.rau.edu.uy

www.universidad.edu.uy

www.elpais.com.uy

www.neticoop.org 
